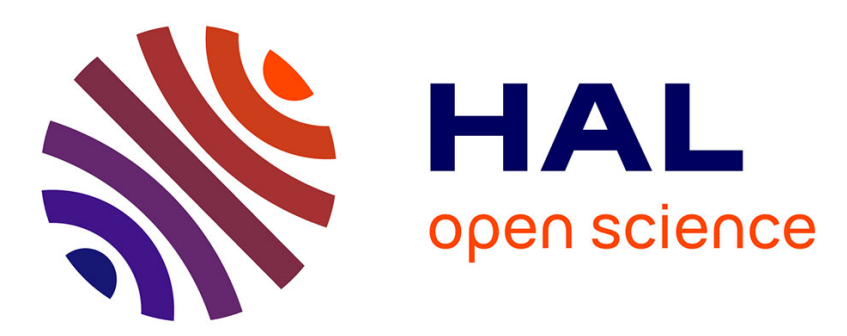

\title{
Modeling the Effects of Maintenance on the degradation of a Water-feeding Turbo-pump of a Nuclear Power Plant
}

Piero Baraldi, M. Compare, A. Despujols, Enrico Zio

\section{- To cite this version:}

Piero Baraldi, M. Compare, A. Despujols, Enrico Zio. Modeling the Effects of Maintenance on the degradation of a Water-feeding Turbo-pump of a Nuclear Power Plant. Proceedings of the Institution of Mechanical Engineers, Part O: Journal of Risk and Reliability, 2011, 225 (2), pp.169-183. 10.1243/1748006XJRR336 . hal-00658486

\section{HAL Id: hal-00658486 \\ https://hal-centralesupelec.archives-ouvertes.fr/hal-00658486}

Submitted on 27 Jul 2012

HAL is a multi-disciplinary open access archive for the deposit and dissemination of scientific research documents, whether they are published or not. The documents may come from teaching and research institutions in France or abroad, or from public or private research centers.
L'archive ouverte pluridisciplinaire HAL, est destinée au dépôt et à la diffusion de documents scientifiques de niveau recherche, publiés ou non, émanant des établissements d'enseignement et de recherche français ou étrangers, des laboratoires publics ou privés. 


\title{
Modeling the effects of maintenance on the degradation of a water-feeding turbo-pump of nuclear power plant
}

\author{
Piero Baraldi ${ }^{1}$, Michele Compare ${ }^{1}$, Antoine Despujols ${ }^{3}$, Enrico Zio ${ }^{1,2}$ \\ ${ }^{1}$ Energy Department, Polytechnic of Milan, Milan, Italy \\ ${ }^{2}$ Ecole Centrale Paris, Supelec, Paris, France \\ ${ }^{3}$ EDF R\&D, Chatou, France
}

\begin{abstract}
This work addresses the modeling of the effects of maintenance on the degradation of an electric power plant component. This is done within a modeling framework previously proposed by the authors, whose distinguishing feature is the characterization of the component living conditions by Influencing Factors (IFs), i.e., conditioning aspects of the component life that influence its degradation.

The original Fuzzy Logic (FL)-based modeling framework includes maintenance as an IF; this requires to jointly model its effects on the component degradation together with those of the other influencing factors. This may not come natural to the experts who are requested to provide the if-then linguistic rules at the basis of the fuzzy model linking the IFs with the component degradation state. An alternative modeling approach is proposed in this work, which does not consider maintenance as an IF that directly impacts on the degradation but as an external action that affects the state of the other IFs. By way of an example regarding the propagation of a crack in a Water-Feeding Turbo-Pump (WFTP) of a nuclear power plant, the approach is shown to properly model the maintenance actions based on information that can be more easily elicited from the experts.
\end{abstract}

Keywords: Maintenance, Degradation Model, Influencing Factors, Fuzzy Logic.

\section{INTRODUCTION}

The significant economic impact of maintenance has led to a strong interest in developing models to support decision makers in their tasks of improving system availability, preventing the occurrence of accidents and reducing maintenance costs of deteriorating systems. The output provided by these models are the values of key parameters used to define the optimal maintenance strategy in the face of various types of maintenance plan and other constraints such as safety requirements and budget limitations.

The effectiveness of the models for supporting maintenance decisions increases when these are able to capture the specificity of the components, which derives from the particular 'life' (failures, shocks, preventive maintenance actions, unavailability periods, work load profile, etc.) that each of them has experienced [1]. For example, in the electrical industry two transformers of the same electrical network installed one on the Alps and one close to the Mediterranean Sea experience very different operating and ambient conditions. For these reasons, the more specifically characterized are the operating and ambient conditions in the model, the more informed can be the supported maintenance decisions. 
The issue of taking into account the influence of covariates representing operating and ambient conditions on the evolution of the degradation process of a component has been addressed in a number of works (e.g., [2]-[4]), also from the theoretical point of view (e.g., [5], [6]). However, as remarked in [7], there are few works (e.g., [7]-[9]) that focus on the influence of covariates in the modeling of degradation processes for maintenance optimization. Furthermore, the stochastic models proposed in these works rely on a number of parameters which may be difficult to estimate in real applications due to lack of real/field data collected during operation or properly designed tests. Indeed, in practice expert judgement is often the main source of information for these models.A novel framework (in the following referred to as 'reference modeling framework') has been proposed ([10]-[11]) to assess the effectiveness of a maintenance policy by modeling the evolution of the degradation mechanisms taking into account the operating and ambient conditions experienced by the component during its life. Within a Condition Based Maintenance (CBM) framework, a maintenance action is performed when the index of the component degradation reaches a predefined limit threshold.

The operating and ambient conditions are characterized by a set of IFs, i.e., conditioning aspects of the component life such as Environment, Operational Mode, Quality, etc. IFs are covariates of the degradation process. The modeling is based on Fuzzy Logic (FL) to deal with the scarce and qualitative information available. The expert knowledge on the influence of the living conditions on the component degradation is represented in terms of if-then-else rules linking the linguistic concepts qualitatively describing the IFs (antecedents) to those describing the degradation state (consequent), e.g., if Environment is Soft and Age is Young then Degradation State is Good'. In the reference modeling framework, maintenance is an IF itself and its effects on the component degradation are modeled jointly with those of the other influencing factors. This leads to numerous antecedents in the if-then linguistic rules which link the IFs with the component degradation state. from the complexity of this rule structure can render difficult the elicitation of the rules by the experts. To overcome this problem, in this paper maintenance is taken out from the set of IFs by applying the concept of imperfect repairs i.e., maintenance actions that partially restore the health state of the component.

Imperfect repairs have been widely investigated in the literature (e.g., [2], [7], [12]-[22]). A classification of the imperfect maintenance models into the two following classes has been proposed in [13]:

i). Models in which the maintenance actions reduce the hazard rate (e.g., Arithmetic Reduction of Intensity (ARI) models of [15], [20] and related references);

ii). Models in which the maintenance actions impact on the effective age or virtual age (e.g., [2], [7], [14], Arithmetic Reduction of Age (ARA) models in [15]).

A further class:

iii). Models in which the maintenance action impacts directly on the degradation level (e.g., [15], [19]-[20]),

is here considered.

The models of class i) cannot be applied in the reference modeling framework since they require that the analytical expression of the hazard rate is known. On the contrary, in the reference modeling framework the failure rate depends on the covariate 'degradation level', whose behavior is influenced by 
the stochastic behaviors of the IFs. This implies the covariate 'degradation level' is a stochastic process, whose analytical expression is unknown. Moreover, the degradation level is an internal covariate since its behavior is influenced by the failure of the component under study, and thus its path carries direct information on the previous stochastic failure history. Due to this fact, in case of 'internal' covariates, the well known exponential survival formula could be no longer valid ([3], [5] and [6]). For example, consider a component restored after a failure; not only its degradation level is reset to the lowest level, but also some IFs (such as Age) or some variables related to the IFs (such as the accumulated vibrations) are consequently modified. This is a further reason to avoid the use of this kind of models when accounting for living conditions.

The basic assumption of virtual age models (class ii) is that at any time instant, the failure rate of a component can be calculated from the failure rate of the component working in nominal conditions (intrinsic failure rate) by considering a virtual age that accounts for the events having caused any rejuvenation or anticipated aging. Thus, in these models the calendar time since the component was firstly operated is substituted for the virtual age. Although virtual age models presented in the literature require assumptions on the analytical expression of the intrinsic failure rate (as the models of class $i$ ), this way of modeling maintenance effects can be applicable to the reference modeling framework by assuming that rejuvenations and modifications of the aging rate due to maintenance actions modify the IFs and thus the evolution of the degradation process.

The models of class iii) focus on the description of the evolution of the degradation processes by means of stochastic processes; the effect of maintenance actions is described by means of improvement functions which define the amount of accumulated degradation that is removed from the component when it undertakes a maintenance action. This class of models is not directly applicable in the present framework. In fact, in order to be as close as possible to the industrial practice, where experts usually classifies the degradation state of a component into a small number (e.g., 3, 4) of discrete states (being this classification based on qualitative measures of symptoms), the degradation process has also to be described by means of a small number of discrete states. The application of a model of class iii) within a modeling of the degradation process in few discrete states leads to consider jumps from one degradation state toward a lower one (e.g. from degradation state 3 towards 2). This may result in an overestimation of the effectiveness of the maintenance actions. On the contrary, in the models of class iii) the improvements of the maintenance actions do not necessarily force the degradation processes to large jumps, since in general there are many, even continuous degradation levels.

The class ii) maintenance modeling approach is developed in this work and compared to that adopted in the reference modeling framework.

The paper is organized as follows: a brief description of the reference modeling framework is provided in Section 2; Section 3 shows the characteristics of the new approach to modeling maintenance and compares it to the original approach adopted in the reference modeling framework; Section 4 describes the case study on which the proposed methodology is applied; finally, some conclusions are given in the last Section.

\section{A brief description of the modeling framework}


The reference modeling framework is partially derived from [23], where a pragmatic approach is proposed to taking into account the component specific living conditions (e.g., environment, working cycles, etc.) by multiplying the base value of the component failure rate by empirical factors. Despite its pragmatism, this approach is not directly applicable in a CBM context which requires the knowledge (even qualitative) of the component degradation level in order to define the most opportune maintenance policy. On the contrary, the approach proposed in the reference modeling framework focuses specifically on the modeling of the degradation process affecting the component, taking into account the actual living conditions in which it works.

Figure 1 gives a snapshot of the modeling framework, which is based on three modules:

- $\quad$ Central Module (CeM); it defines the IFs that actually influence the considered degradation mechanism.

- $\quad$ Backward Module (BM); the physical variables related to each IF are identified, and the relationships between them and the IF are determined.

- $\quad$ Forward Module (FM): the link between the IFs and the degradation process is defined. The degradation process is described by means of a small number of levels, or degradation 'macro-states', each one characterized by a failure rate. The choice of this representation is driven by industrial practice: experts usually adopt a discrete and qualitative classification of the degradation state based on qualitative interpretations of symptoms.

Eliciting information from experts, resorting to the literature, inferring from databases etc. are different ways to address the contents of these modules.

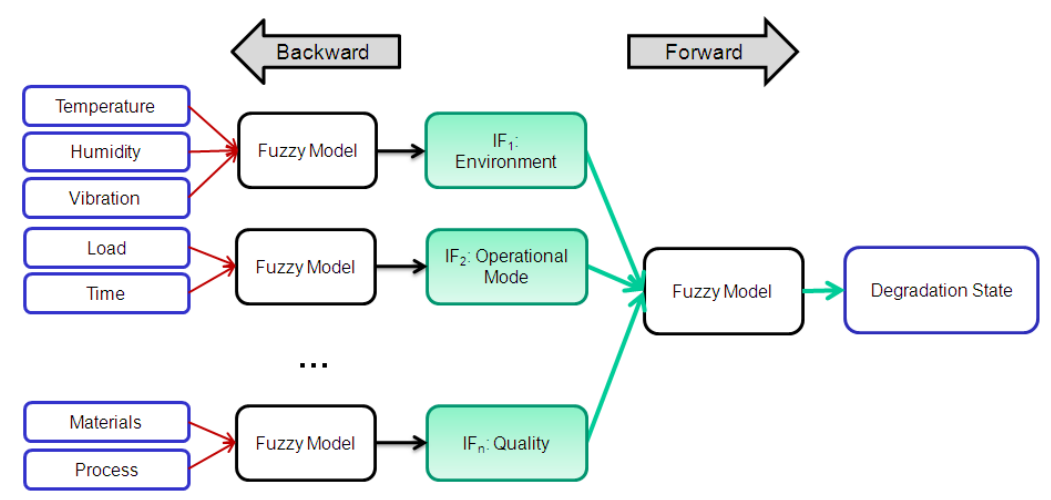

Figure 1: snapshot of the degradation modeling framework.

Both the BM and the FM are developed by applying FL theory to cope with the scarcity of the data typically available and its qualitative nature. In practice, the IFs are expected to be more easily represented by linguistic variables rather than numeric variables (e.g., 'the environment is mild' or 'the maintenance is efficient'). In this case, fuzzy logic offers the capability of dealing with imprecise variables and linguistic statements provided by experts on the basis of their knowledge and engineering sense of practice.

Furthermore, the typically stochastic behavior of the living conditions results in randomness of the covariates/IFs, and thus stochastic transitions between the degradation levels (and associated values of failure rates).

The degradation model can be used to test the effectiveness of a maintenance policy. To do this, the degradation stochastic evolution is simulated by the model and the failure rates associated to the 
degradation levels evolving in time are input to a Monte Carlo (MC) module which estimates the availability of the system over a specified time horizon (Figure 2); through a cost model, the total costs associated to the maintenance policy can then be assessed [10]-[11].

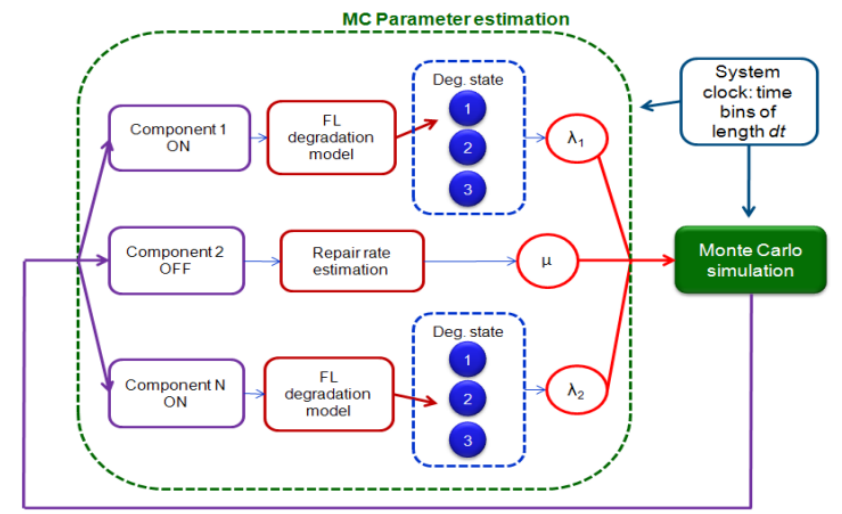

Figure 2: interface between FL degradation and MC simulation models.

\section{Maintenance Modeling approach}

In the reference modeling framework, maintenance is considered as an IF. The physical variables (e.g., frequency of the inspections, accuracy of maintenance actions, number of maintenance actions overtaken by the component, etc.) on which this IF depends are identified and a Fuzzy Rule Base (FRB) is built to combine them to the Maintenance level. For example, a rule of this FRB may be: 'if Inspections are Frequent and Maintenance Action Accuracy is Good and Number of Maintenance Actions is Low then Maintenance is Good'.

The Maintenance IF is an antecedent of the rules of the FRB that describes the relation between all the IFs (e.g., Environment, Maintenance and Age) and the degradation level of the component. For example, a rule of this FRB could be: 'if Environment is Mild and Maintenance is Good and Age is Young then Degradation State is Good'.

Once the fuzzy models have been built, they can be used for the identification of the degradation state of a component which has experienced given operational and ambient conditions. The Fuzzy Inference System adopted is of Mamdani type ([29], [30]).

In such modeling framework, the impacts of a maintenance policy on the degradation process need to be evaluated jointly with the effects of the other IFs. This may be difficult for experts that need to build rules on the basis of multidimensional antecedents and account for numerous aspects specific to maintenance (e.g., effects of actions like lubrication and cleaning, different effectiveness of maintenance actions during the component life, sensitivity of the degradation dynamics to the inspection period) which may influence the other IFs.

In the present work, an alternative modeling choice is investigated: maintenance does not influence the degradation process directly, but it is taken into account by modeling its effects on the physical variables which the other IFs depend on (Figure 3 and Figure 4). Thus, the expert has to identify the effect 
of each maintenance action on these variables. For example (Figure 4), the maintenance actions can impact on the variables Age Setback and Environmental Quality which are input variables of the BM of the IFs Age and Environment, respectively. A rule for describing the influence of the maintenance on the Age Setback may be: 'if Number of Overtaken Maintenance Actions is Small and Calendar Time Since Last Maintenance Action is Medium and Current Degradation Level is Good and Quality of Maintenance Actions is Good then Age Setback is Large'; a rule that links maintenance to Environmental Quality may be with the same antecedents and 'Environmental Quality is Good' as consequent.

With regards to the modeling complexity, if compared to the approach presented in the reference modeling framework, the present approach has one less IF (i.e., the Maintenance) and thus requires the elicitation of a smaller number of rules for the constitution of the FRB of the Forward Module. On the other side, building the maintenance model of Figure 4 requires to model the effect of maintenance on the other IFs and thus may require additional variables in input to the BM modules of the IFs.

As highlighted in [14], an important issue that the maintenance modeling should take into account is aging: the degradation level of a component will unavoidably tend to increase even if maintenance actions are performed regularly and neatly. This issue is here addressed by modeling age setbacks that decrease over time even if the same maintenance action with the same care is performed during the component lifetime and imposing that there exists a part of the life in which these setbacks are not sufficient to reset component age to zero. In the maintenance modeling approach proposed in the reference modeling framework the expert is requested to build rules that implicitly account for the different efficiency of the maintenance actions during the component life time.

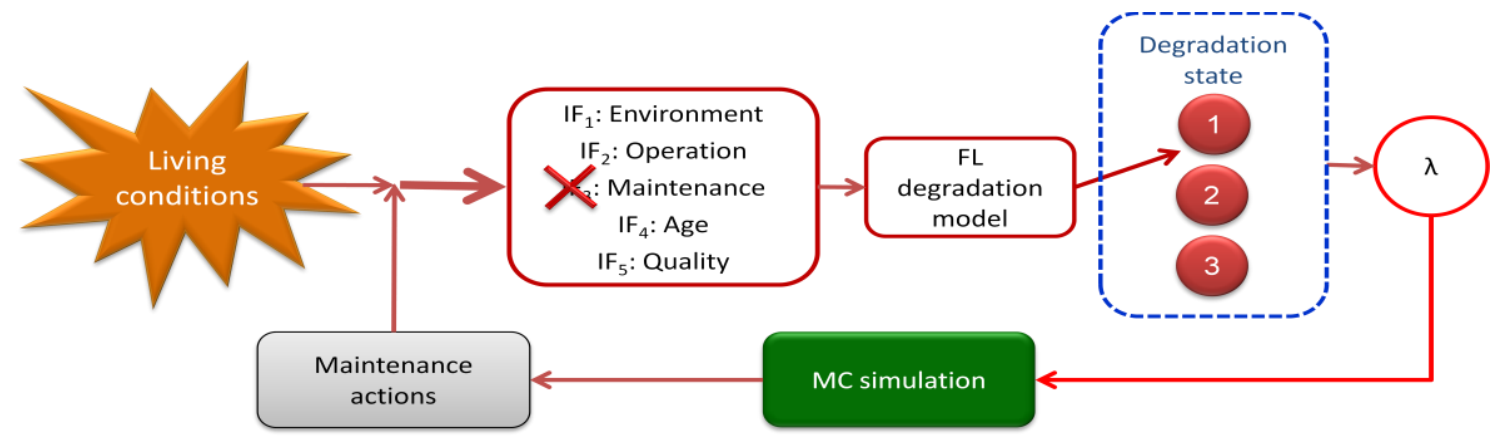

Figure 3: modeling of the maintenance effects.

In [24] it has been noted that there are maintenance actions, as for example lubrication and cleaning, that do not result in age setbacks, but in changes of the aging rate. These maintenance actions are nothing but improvements of the environment in which the component works; thus, in the proposed approach they are taken into account by the rules that describe their influence on the IF 'Environment' (see Figure 4). Again, the modeling choice implemented in the reference modeling framework requires that when building the FRBs, experts take into account that some of the maintenance actions that will be performed during the component time horizon are lubrications, cleaning, etc and thus have effects different from other PM actions. This may be very difficult in practice.

It is also interesting to take a glance at how the maintenance models of the literature address the issue of modeling maintenance actions like lubrication and cleaning. The pure ARA and ARI models investigated in [15] are not suitable to modeling these actions, whereas extended ARI and ARA approaches (e.g. [2], 
[22]) are able to account for them by adjusting the aging rates when components overtake these maintenance actions. Also the models of class iii) in which maintenance actions impact on the degradation levels can model this type of maintenance actions, for example by considering stochastic models that change their parameters at the occurrence of maintenance events (e.g. a correction factor applied to the shape parameter of a gamma process that decreases the propagation speed for a given time interval; in [19], a change in the shape parameter is accompanied with a jump of the degradation level).

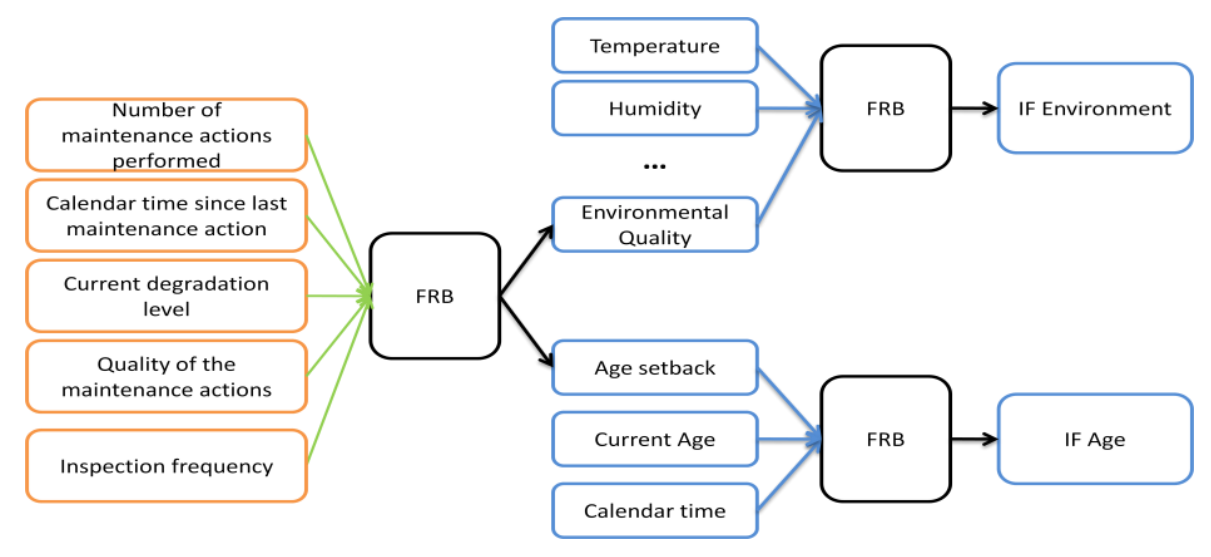

Figure 4: modeling of the influence of maintenance actions on the IFs.

A further comparison between the proposed maintenance modeling approach and other approaches of literature concerns the way in which stresses caused by the living conditions are taken into account. In the proposed approach they are not captured only by the IF Age, but also by the other IFs; this modeling choice differs from that adopted in [2] where stresses are modeled by modifying only the relation between age and chronological time; thus, the IF Age accounts for both the living conditions and impacts on the evolution of the failure rate.

\section{Case study}

In order to illustrate the application of the proposed methodology, an example concerning a WaterFeeding Turbo-Pump (WFTP) of a steam generator of a nuclear power plant is considered in this Section. In particular, this example is derived from a real case study investigated by Electricité de France (EDF) experts, who have identified by means of a Failure Mode and Effect Analysis (FMEA) the degradation processes affecting the component and the associated IFs and symptoms. These latter are defined as consequences of the degradation process that are observed by the operators during the component inspections. For example, vibrations and over-heating may be the symptoms associated to the degradation of the teeth of a gear. Fatigue degradation mechanism is revealed to be one of the most critical processes affecting the component and thus it has been chosen as case study in the present work. Notice that no consideration has been given to other degradation mechanisms or components although, as stated by the EDF experts, some may lead to an acceleration of the degradation process under consideration and in some cases even to the failure of the considered device (cascade effect). The ability of the framework to model multi-components system is an open issue to be addressed. 
In the considered case study, the experts have been involved only in this preliminary investigation and not in the effective development of the degradation model. The information necessary for the development of the proposed degradation model has been acquired by using a physical model of the degradation process. Notice that in practical cases the degradation model is not expected to be available and one can resorts only to expert knowledge for the development of the fuzzy degradation models. In this work, the use of a physical model has allowed us to verify what is the information necessary for the model development and if the obtained model can be effectively used for maintenance policy assessment. In particular, the physical model has been used to simulate some component degradation evolutions corresponding to different living conditions in order to extract the fuzzy rules of the fuzzy degradation model. Furthermore, the physical model parameters, which are usually unknown or uncertain, are supposed to be exactly known.

The degradation of the considered component due to fatigue is caused by the development of cracks. The creation and propagation of these cracks is a complex physical phenomenon, which has been modeled in a number of different ways (e.g.,[25]-[27]). According to these models, the degradation is mainly influenced by the loads applied on the component, its constitutive materials and production process and some geometrical factors characterizing the crack such as its size, notch radius, position with respect to the direction of the loads, etc.

In this work, it is assumed that the length of the most critical crack of the component defines its degradation level and that this length can only increase in time; in other words, PM actions on the crack cannot shorten it.

In the modeling, the following three degradation states are considered (see also Figure 2):

1. Good: the component is as new or almost new; no maintenance actions are foreseen if the component is in this state.

2. Medium: the component in this state needs some actions aimed at decreasing the crack growth speed.

3. Bad: if the component is in this degradation state it is convenient to replace it.

To each degradation state, the failure rates reported in Table 1 have been associated. Their values can be determined from real plant data, if available, or from expert knowledge. In this respect, the degradation states observed at inspection and the component failure times are information that can be collected and used to estimate the failure rates associated to the different degradation states.

Finally, notice that although the failure rates associated to the degradation states are constants, the component experiences an increasing failure rate during its life, since its degradation state evolves from 'Good' to 'Bad' until failure. Thus, the component failure rate can be seen as a stepwise function whose steps have values, and.

Table 1: Failure rates

\begin{tabular}{|l|l|}
\hline Degradation State & Failure rate \\
\hline Good & \\
\hline Medium & \\
\hline Bad & \\
\hline
\end{tabular}

The CBM policy applied to the system is composed by the following tasks: 
- Inspection: this action, aimed at detecting the degradation state of the component, is considered to be of negligible duration but has a cost of $€$. Furthermore, this is the only scheduled action.

- CBM actions: PM actions which are dependent on the result of an inspection action. If the component is found to be in state "Good", no action is performed. If the degradation state is "Medium", the component undergoes a repairing action aimed at slowing down the degradation process: this action has a duration of and a cost of $€$. Finally, if the component is in state "Bad", it is replaced: this action takes of time and costs $€$.

- Corrective Maintenance (CM) actions. The corrective action, performed after a component failure, is assumed to be the replacement of the component. Due to the fact that this event is unscheduled, this action brings an additional duration of and an additional cost of $\quad €$, with respect to the replacement after an inspection, leading to a total duration of and to a total cost of $€$. In particular, the additional time may be caused by the supplementary time needed for performing the procedure of replacement after failure or to the time elapsed between the occurrence of the failure and the start of the replacement actions.

Both the amplitude and the frequency of the fundamental wave, which constitute the measurable variables in input to the BM of the IF environment, are assumed to change according to a Compound Poisson Process (CPP, . [3] and [28]). This modeling choice is justified by considering that the vibration in the location in which the component of interest works is caused by other components due to their degrading (e.g., the increase of the eccentricity of the center of gravity in rotating machines) or to the plant design, which results in the periodic application of a load on coupled components (e.g., alternating machines discharging loads on the same basement of the component of interest). Since, in general, the behavior of the components producing the vibration is stochastic, the vibration profile suffered by the components is also stochastic. In the present case study, it has been supposed that the external events that cause modifications both in the amplitude of the wave (e.g., failures that modify the eccentricity of rotating systems and relevant maintenance actions) and in its frequency (e.g., increasing/decreasing of the operational load of a vibrating system) occur randomly with a given rate, whereas the amount of the changes is uniformly distributed. This leads to consider a CPP process whose parameters are:

- the time of modifications of both the amplitude and frequency of the fundamental wave, which is assumed to be exponentially distributed, with parameter

- the new frequency value which is assumed uniformly distributed in the range

- the new amplitude value which is assumed to be uniformly distributed in the range

\subsection{Physical model of the degradation process}

In this paragraph, the Paris-Erdogan model (one of the best known physical models in fracture mechanics) is briefly presented. The fatigue crack growth can be modeled as a process divided into three different parts (Figure 5):

- Initiation (or incubation): the period during which the crack has not yet appeared. This step ends at a time and corresponds to the 'Good' degradation state of the component.

- Propagation: the crack has already appeared (i.e., it has reached the detection threshold, ) and is growing slowly until it reaches a critical size, . This part corresponds to the 'Medium' degradation state of the component. 
- Rupture: after the achievement of the critical size, , the largest crack reaches a faster growth and leads to the failure of the component. This part corresponds to the 'Bad' degradation state of the component.

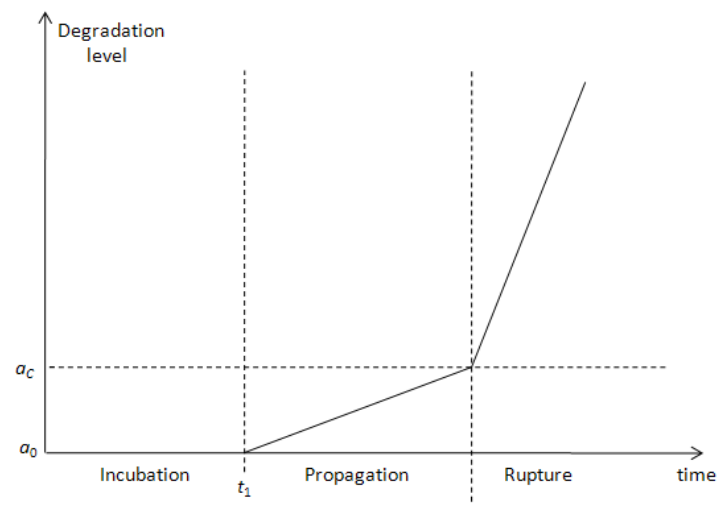

Figure 5: fatigue crack growth model.

The Paris-Erdogan model relates the increment of crack growth per cycle, , to the parameters of stress range, , and instantaneous crack length, :

where and are constants determined by material properties and is the fluctuation range of the crack tip stress-intensity factor , which depends on the size and the type of the crack [27]. A simple model for is given by:

where is the stress at the crack tip and is the geometry correction factor which depends on the crack shape, length and on the component shape. A backward difference approximation can be obtained as:

where

According to this model, the crack growth accumulates relatively slowly and continuously with the load cycles . In this case study, the stress range, , has been linked to the amplitude, , of the fundamental wave by means of the constant parameter , which, for the sake of simplicity and without loss of generality, has been assumed equal to 1 :

Table 2 reports the values of the parameters of the Paris-Erdogan model that have been used in the considered case study. Notice that the parameters values used in this case study are not derived from a real application; they have been arbitrarily assumed for the purpose of illustration. 
Table 2: parameters of the Paris-Erdogan model used in the case study.

\begin{tabular}{|l|l|}
\hline Parameter & Value \\
\hline & \\
\hline & \\
\hline & \\
\hline & \\
\hline & \\
\hline & \\
\hline
\end{tabular}

\subsection{Fuzzy model of the degradation process}

The evolution of the degradation process is assumed to be dependent on two IFs:

- $\mathrm{IF}_{1}$ : Environment. The influence of the environment on the considered degradation mechanism is assumed to be mainly caused by the vibrations in the location in which the component works. In particular, the measurable variables on which the $\mathrm{IF}_{1}$ depends are the mean values of the frequency and of the amplitude of the vibration fundamental wave in the time elapsed since the component has started to work. The UoD of this IF, arbitrarily scaled on , is partitioned into three Fuzzy Sets: 'Soft', 'Medium' and 'Heavy' (Figure 6, left) whose membership functions (MFs) achieve the maxima in correspondence of $0,0.5$ and 1 , respectively. For the sake of simplicity, the MFs are assumed to be linear and to sum to 1 in any point of the UoD.

- $\mathrm{IF}_{2}$ : Age. This IF represents the virtual age of the component. The UoD of this IF is the interval , where

is the time horizon; on this interval, three Fuzzy Sets 'Young', 'Medium' and 'Old' are defined by means of triangular membership functions (Figure 6, right). In particular, the component is considered 'Young' when it has no visible crack. This is completely true at the beginning of the component life (engineering good sense suggests that a brand new component has no cracks) whereas it is completely false after (the physical model simulations show that no component works for more than without any crack having appeared). On the opposite, after the component is considered 'Old' with membership 1: simulations of the physical model prove that no component is able to work for more than The MF of level 'Medium' is assumed to be the complement to one of the sum of the MFs of the other two levels, which for simplicity are assumed to be linear.

\subsubsection{Model of the maintenance action effects}

In this case study, for the sake of simplicity, modeling of maintenance actions is not addressed within the FL framework but it is assumed that the value of the IF/covariate Age, , changes when a repair action is performed according to: 
where is the number of repair actions already performed on the component. Notice that a deterministic model of the maintenance action effects is not known in practical cases where the information available is expected to come from expert knowledge. However, using a deterministic model of the effects of the maintenance actions allows to compare the results obtained by the fuzzy degradation model with those of the application of the physical degradation model of Section 4.1.
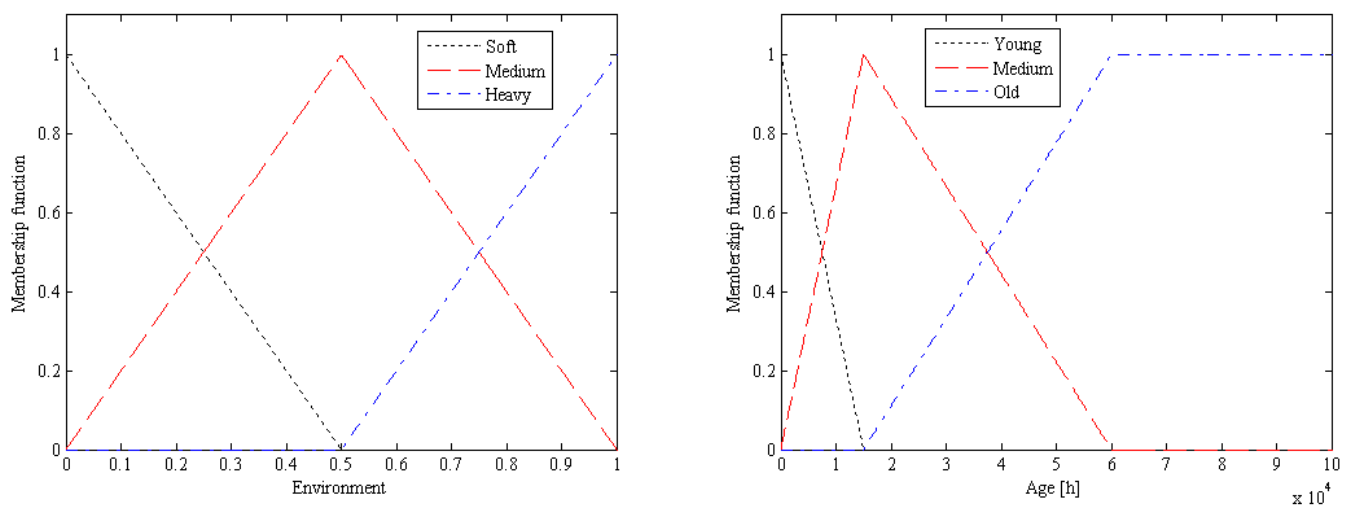

Figure 6: fuzzy sets of the IFs: Environment (left) and Age (right).

\subsubsection{Backward Module}

$\mathrm{IF}_{1}$ depends on two physical variables which can be measured by means of sensors (e.g., strain gauges): the amplitude and the frequency of the vibration fundamental wave. In particular, the mean values of these variables in the time elapsed since the system has started to work are given in input to the BM. Figure 7 shows the fuzzy sets, defined by means of trapezoidal membership functions, partitioning the variables in input to the BM. In particular:

- The mean value of the frequency of the fundamental wave is described by the fuzzy sets "Low", "Medium" and "High", defined on the UoD

- The mean value of the frequency of the fundamental wave is described by the fuzzy sets "Low", "Medium" and "High", defined on the UoD

The UoDs and the MFs of the fuzzy sets have been set by using expert judgement.
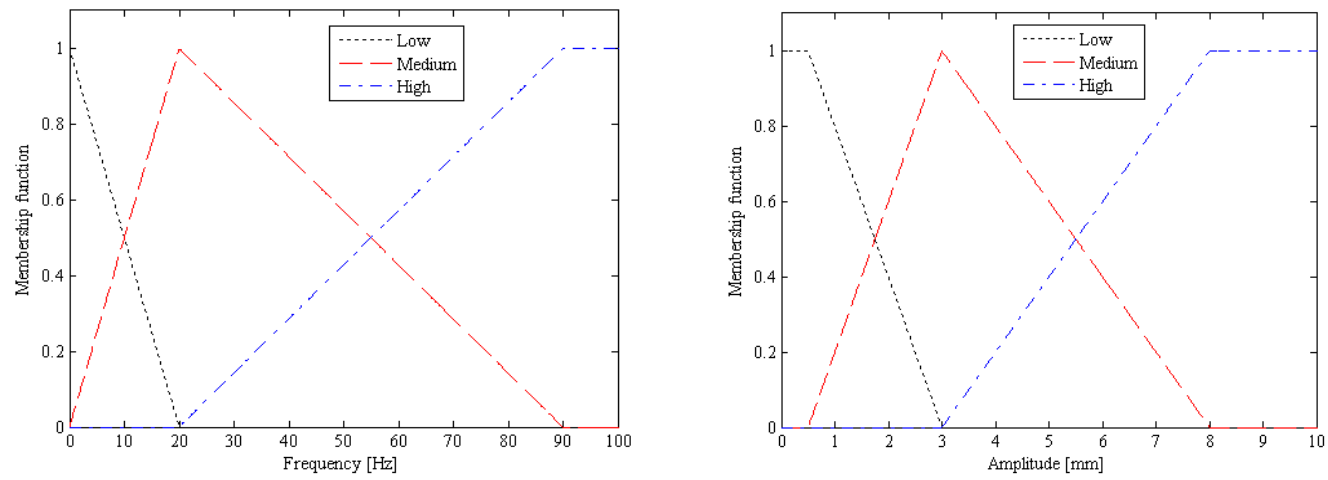

Figure 7: fuzzy sets of Frequency (left) and Amplitude (right). 
Table 3 reports the rules that combine the two inputs of the BM module and thus evaluate the quality of the environment in which the mechanical component works. For example, the cell in column 5 and row 5 expresses the rule: 'if Frequency is High and Amplitude is High then Environment is Heavy'.

Table 3: fuzzy rules of the $\mathrm{BM}$ relative to the $\mathrm{IF}_{\mathbf{1}}$.

\begin{tabular}{|c|c|c|c|c|}
\hline & & \multicolumn{3}{|c|}{ Amplitude } \\
\hline & & Low & Medium & High \\
\hline \multirow{3}{*}{ 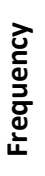 } & Low & Soft & Soft & Medium \\
\hline & Medium & Soft & Medium & Heavy \\
\hline & High & Soft & Heavy & Heavy \\
\hline
\end{tabular}

\subsubsection{Forward Module}

The Forward Module is addressed by setting the rules summarized in Table 4. For example, the cell in row 4 and column 3 defines the rule: 'if Environment is Soft and Age is Young and Previous Degradation State is Good then Degradation State is Good'. Thus, the rules in Table 4 have three antecedents: Environment, Age and Previous Degradation State (PDS); this latter is not an IF and has been introduced in order to ensure that the degradation state does not decrease as the age of the component increases ([10][11]).

Notice that the rules in Table 3 and Table 4 have been defined by simulating with the physical model the different degradation evolutions corresponding to the different environmental conditions and thus by qualitatively extracting the degradation states in the different age levels.

Table 4: fuzzy rules defining the relationship between the IFs and the degradation state.

\begin{tabular}{|c|c|c|c|c|}
\hline & & \multicolumn{3}{|c|}{ Environment } \\
\hline & & Soft & Medium & Heavy \\
\hline & Age & & & \\
\hline \multirow{3}{*}{$\begin{array}{l}\text { PDS: } \\
\text { Good }\end{array}$} & Young & Good & Good & Good \\
\hline & Medium & Medium & Medium & Bad \\
\hline & Old & Medium & Bad & Bad \\
\hline \multirow{3}{*}{$\begin{array}{c}\text { PDS: } \\
\text { Medium }\end{array}$} & Young & Medium & Medium & Medium \\
\hline & Medium & Medium & Medium & Bad \\
\hline & Old & Medium & Bad & Bad \\
\hline \multirow{3}{*}{$\begin{array}{l}\text { PDS: } \\
\text { Bad }\end{array}$} & Young & Bad & Bad & Bad \\
\hline & Medium & Bad & Bad & Bad \\
\hline & Old & Bad & Bad & Bad \\
\hline
\end{tabular}




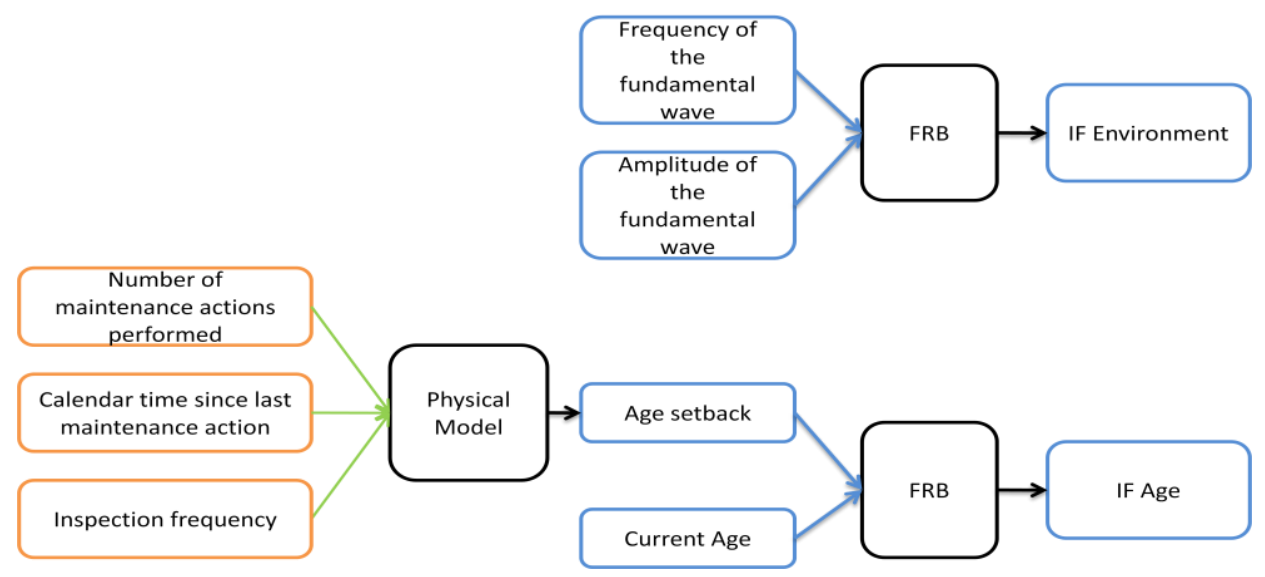

Figure 8: modeling of the influence of maintenance actions on the IFs: tailoring to the present case study.

\subsection{Results and comparisons}

In this Section, the results obtained by applying the modeling approach proposed in this work (referred to as Maintenance as External Effect (MEE)) are reported and compared with the results provided by both the physical model and the approach proposed in the reference modeling framework (referred to as Maintenance IF (MIF)).

Figure 9 shows the mean unavailability of the component for different values of the Inspection Interval, , with the related $68.3 \%$ confidence interval which represent the uncertainty affecting the estimation due to the use of the $\mathrm{MC}$ method for its computation. In this case study, the confidence intervals are very narrow because of the large number $\left(10^{5}\right)$ of $M C$ simulations performed.

Notice that there is a very good agreement between the two fuzzy logic-based approaches, MIF and MEE, whereas a bias with respect to the physical model mean unavailability is observed. This bias depends from a modeling imprecision of the fuzzy models built using only the limited information coming from the observation of some component degradation evolutions. In this respect, notice that if more information were available for the model building, more accurate predictions of the mean unavailability would be obtained.

The mean unavailability computed by the MIF and MEE approaches presents two minima in correspondence to the Inspection Intervals of and . In both approaches, these minima emerge due to the fuzzy rules used to describe the degradation of the component. For example, in the MEE approach, Table 3 shows that the transition from the degradation state 'Medium' to the state 'Bad' in case of environment 'Heavy' is represented by the rules 'if Environment is Heavy and Age is Young and PDS is Medium then Degradation state is Medium' and 'if Environment is Heavy and Age is Medium and PDS is Medium then Degradation state is Bad'. Thus, this transition requires that the age of the component fires with an highest degree of membership the fuzzy set 'Medium' rather than the fuzzy set 'Young', i.e., the age should be at least or Then, for a the probability of detecting the 'Bad' degradation state before the component failure (i.e., the probability of performing a PM action instead of a CM one) is significant since the component will work in the 'Bad' degradation state for only (between and ). In particular, this probability is given by: 


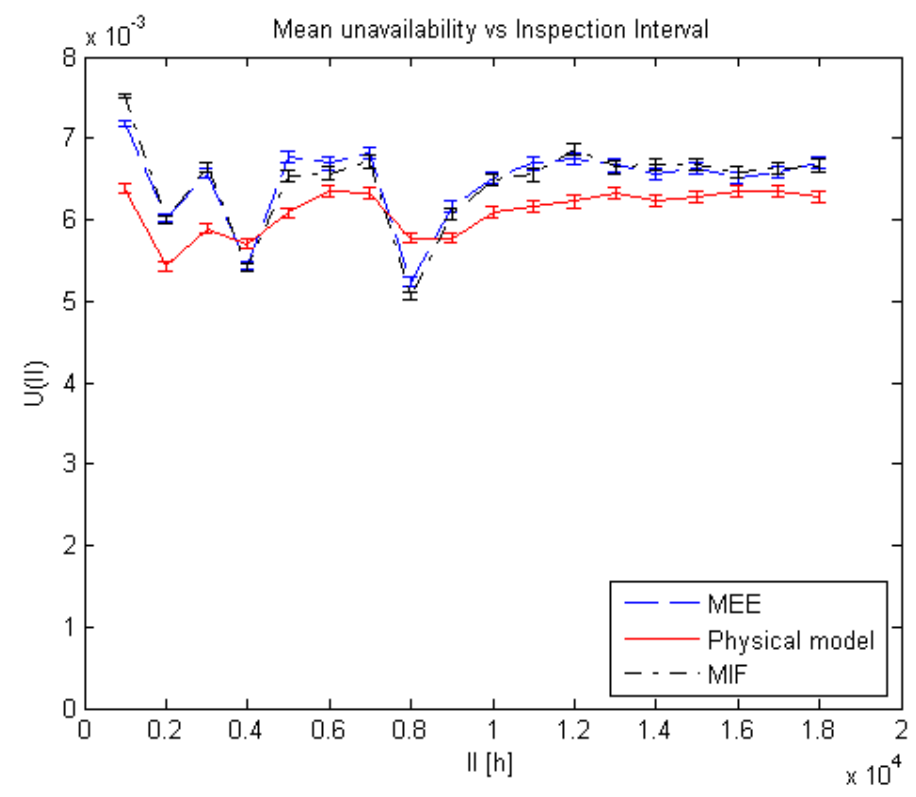

Figure 9: mean unavailability of the component, with the related $68.3 \%$ confidence interval (i.e., one standard deviation), for different values of the inspection interval.

On the contrary, for different inspection intervals, e.g. detect the 'Bad' degradation state is performed later (at or , the inspection task which can and at , respectively); thus, the associated probabilities of detecting the 'Bad' degradation state are:

The effectiveness of the detection and replacement action, which avoids the large down-time corresponding to the unscheduled and potentially dangerous failure of the component, is thus heavily reduced when passing from to or to

To further investigate this aspect, the mean unavailability is decomposed in its contributing parts: Figure 10 reports the mean contribution of the failures (and, thus, of the corrective maintenance), Figure 11 that of the repair actions which follow the detection of the 'Medium' degradation state of the component and finally Figure 12 that of the replacement actions which follow the detection of the "Bad" state. These mean values are reported with the associated $68.3 \%$ confidence intervals. It can be noted that the principal contributions to the mean unavailability are due to failures and, for frequent controls, repair actions, whereas a negligible contribution comes from the preventive replacements. In particular, the contribution of the failure to the unavailability has an increasing trend, even if two local minima are located at and for the reasons explained above. The contribution to the unavailability of the repair actions is decreasing with the increasing inspection interval. This contribution increases in correspondence to an inspection interval of about due to the fact that at timen the Environment is "Soft" or "Medium", the degradation state of the component makes a transition from "Good" to "Medium". Finally, the contribution of the preventive replacement to the unavailability has a 
trend which is decreasing with the increase of the inspection interval; however, as stated above, this contribution can be neglected without a great loss of accuracy.

The bias between the unavailability estimated by the two FL-based approaches and by the physical model is mainly due to the contribution of the failures (see Figure 10): the degradation process of the physical model is not exactly reproduced by the fuzzy logic-based models; in particular, in some situations the fuzzy logic-based model is found to overestimate the degradation level reached by the component. This is mainly due to the small number of fuzzy sets partitioning the IFs (i.e., the Environment and the Age), which forces the analyst to approximate the representation of the degradation process; however, the larger the number of fuzzy sets the larger the number of fuzzy rules, which may be difficult to set up by the expert.

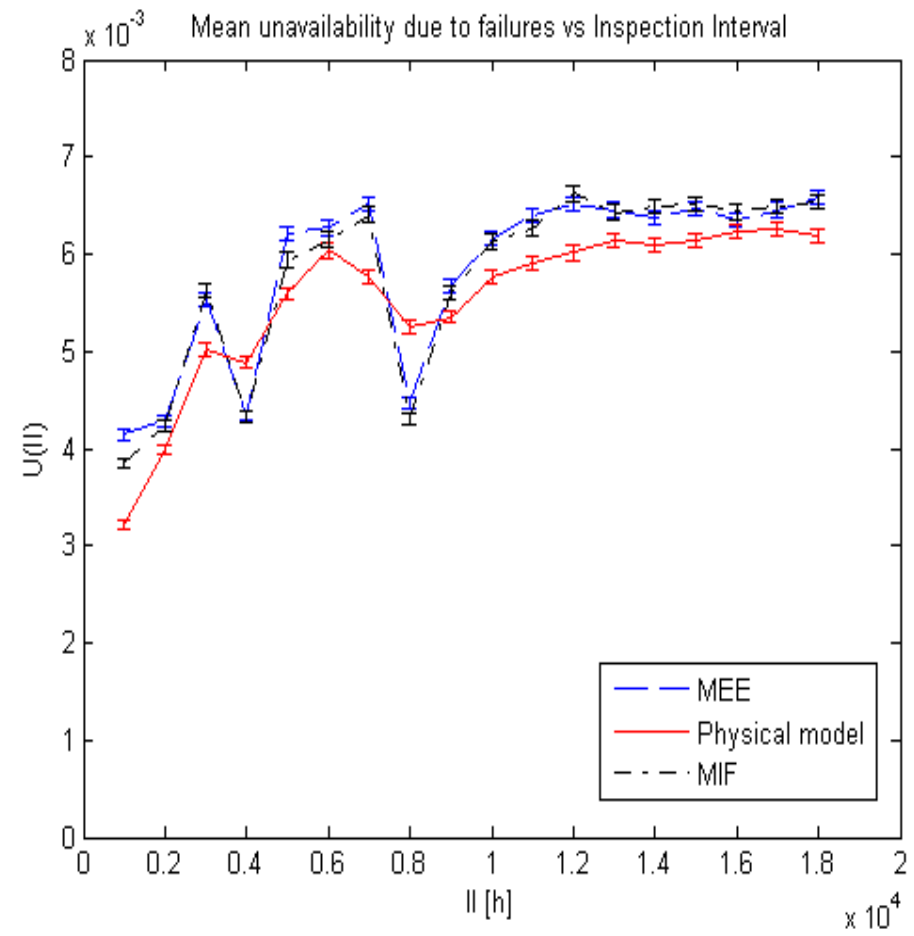

Figure 10: contribution of the corrective maintenance to the mean unavailability. 


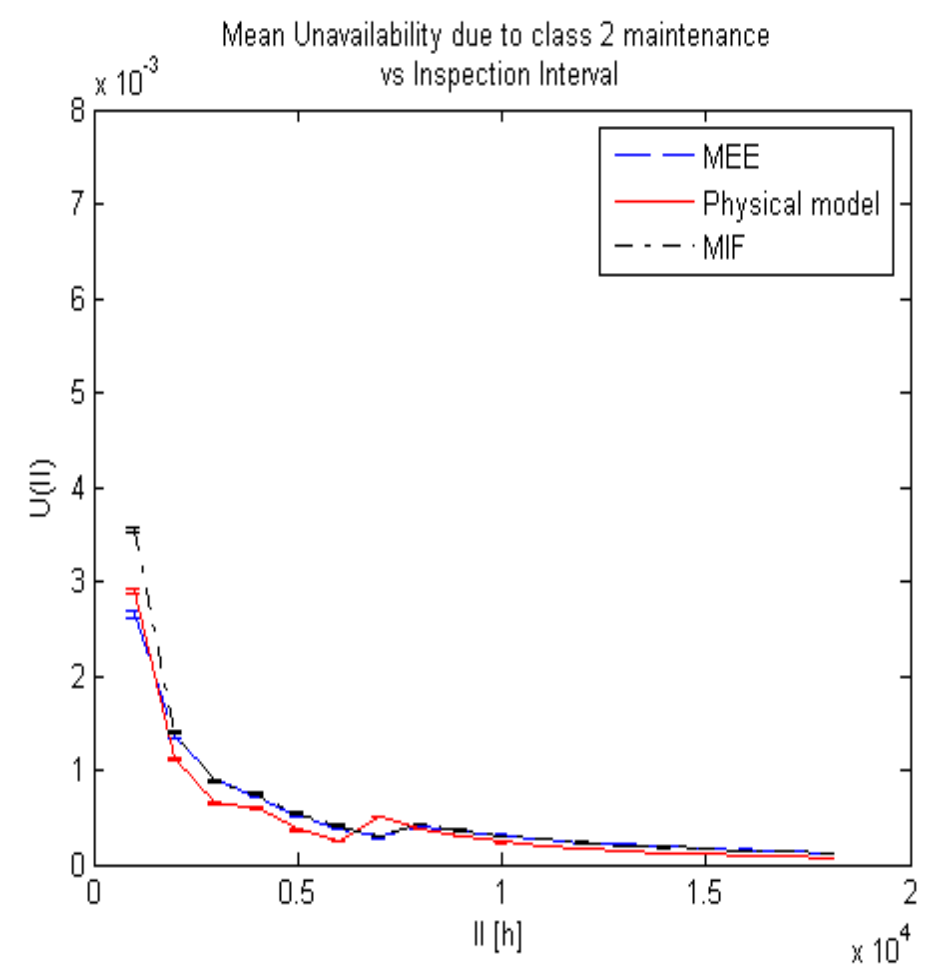

Figure 11: contribution of the PM repair actions to the mean unavailability.

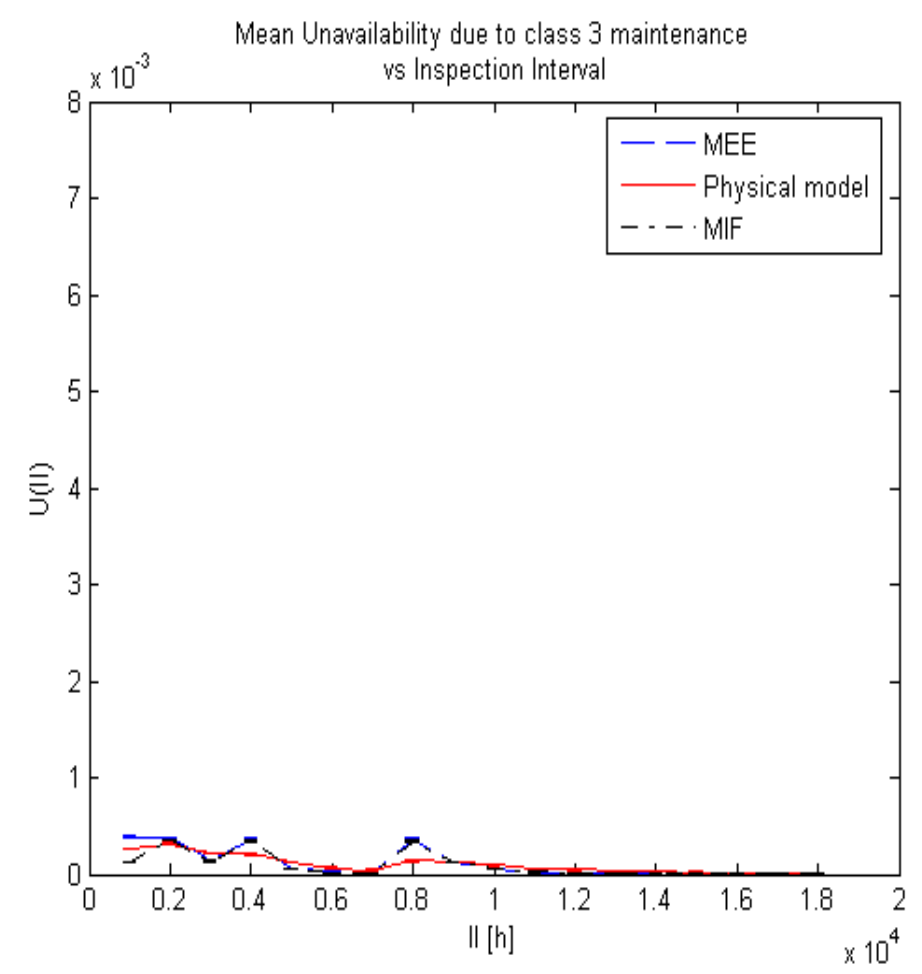

Figure 12: contribution of the PM replacement actions to the mean unavailability.

Figure 13 shows the total costs associated to the maintenance policy of the component when varying the inspection interval; it shows a trend which is similar to that of the mean unavailability. The results found applying the two fuzzy logic-based degradation models are close one another, but a bias is observed with respect to the result of the application of the physical degradation model. This bias has the same cause of that on the mean unavailability estimation (Figure 9). 


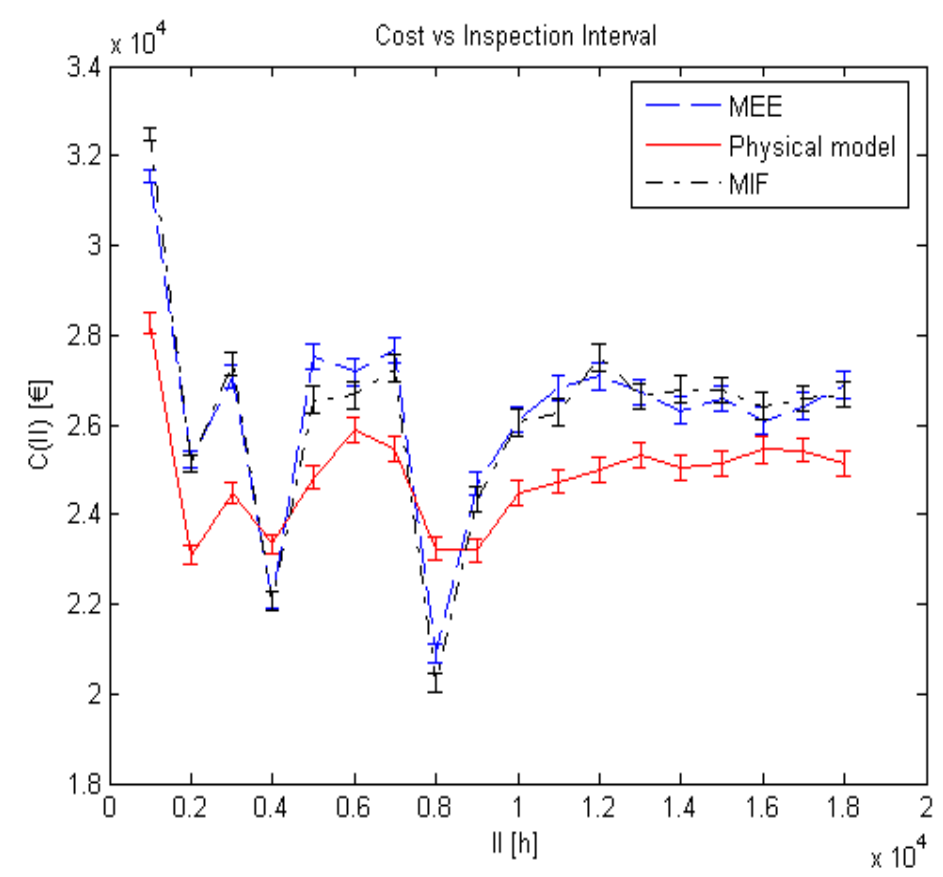

Figure 13: total cost of the component maintenance, with the related $68.3 \%$ confidence interval, for different values of the inspection interval.

A comment is in order about the assessment of the maintenance policy: the physical model based on the Paris-Erdogan law suggests that very rare inspections (i.e., with ) or very frequent (i.e., with ) are inefficient. Furthermore, there is also a range of intermediate inspection intervals (i.e., with ) in which the maintenance is found to be inefficient. Both the MIF and MEE approaches provide results which are consistent with the physical model, although with the bias mentioned above. In particular, the total maintenance cost presents two minima, corresponding to and , which are located, respectively, in the range and in the range , identified by the physical model as the ones in which the total maintenance cost is smaller. This proves the potential of both the MIF and MEE approaches.

Finally, the instantaneous unavailability estimated by both MIF and MEE approaches are reported in Figure 14 and Figure 15 for inspection intervals and , respectively; in particular, these are also compared to the unavailability computed by applying the physical model. It can be observed that there are peaks in correspondence to the multiples of the inspection interval, due to the preventive maintenance performed on the component. The peaks obtained when applying the fuzzy logic-based models are larger; this is due to the fact that the degradation state is slightly overestimated with respect to the physical model and, thus, also the average number of preventive maintenance actions is larger. 

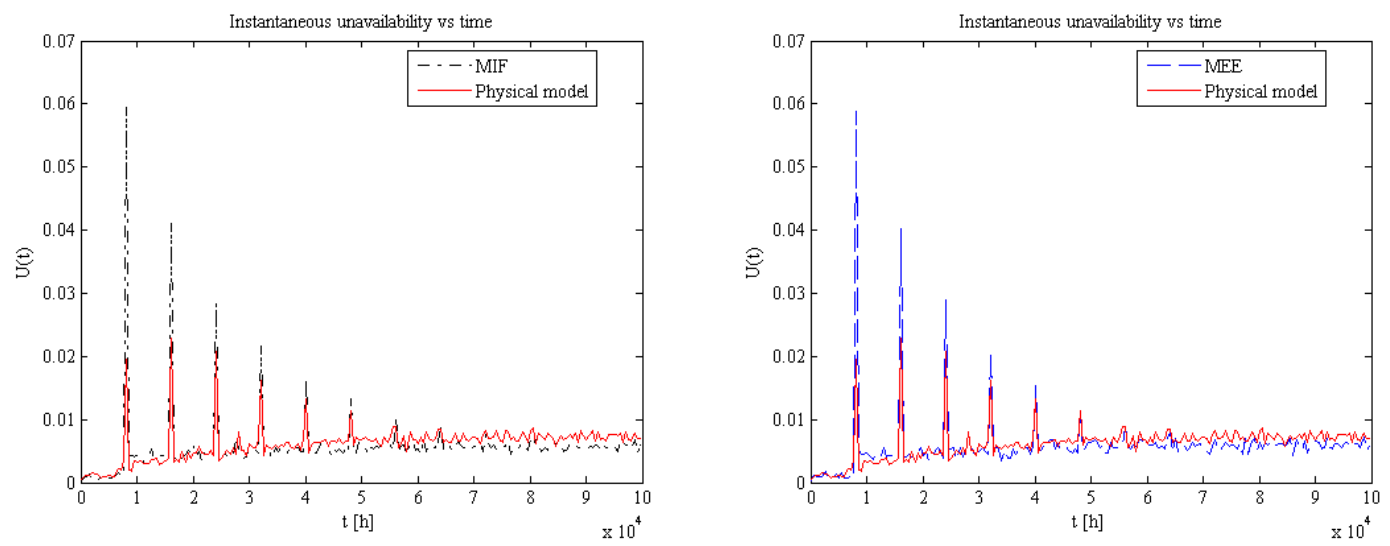

Figure 14: instantaneous component unavailability during the time horizon, with an inspection interval of $8000 \mathrm{~h}$.
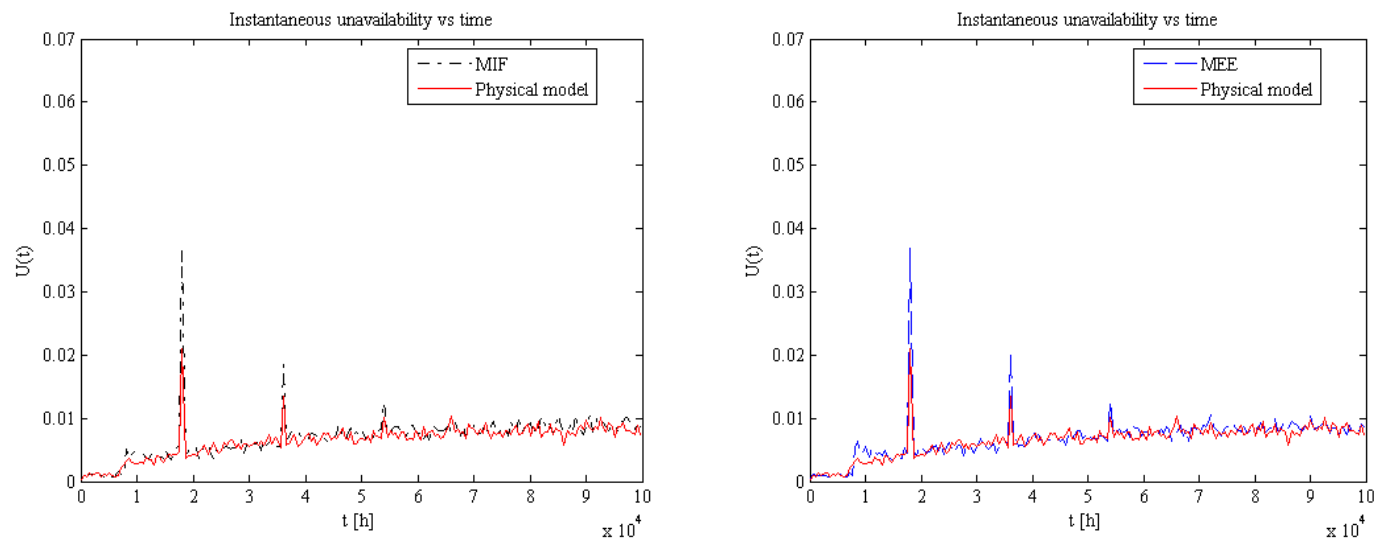

Figure 15: instantaneous component unavailability during the time horizon, with an inspection interval of $18000 \mathrm{~h}$.

\section{Conclusion and Future Work}

An approach for modelling the impact of a maintenance policy on the evolution of the degradation process affecting a component has been proposed in this work. This has been developed within the framework presented in the reference modeling framework and is based on the concept of imperfect repairs. The differences between the maintenance modelling approach here proposed (MEE) and that investigated in the reference modeling framework (MIF) have been pointed out.

A WFTP of a steam generator of a nuclear power plant affected by fatigue degradation process has been considered as case study, and the two modelling approaches have been tailored on it. This has allowed to illustrate in detail the modelling framework and also to compare the MIF and MEE approaches from a practical point of view. A physical model, for describing the actual component degradation behavior, has been applied on the same case study in order to provide the comparison term for assessing the performance of the two models. In practical cases, the physical model and/or its parameters may not be known, and degradation process may only be modeled by resorting to expert knowledge, which is of qualitative nature. The MIF and MEE models capture the knowledge of the experts about the degradation process, and provide results that are affected by the uncertainty deriving from the imprecise modeling made by the experts. In the case study examined, although some differences arise between the physical and the two fuzzy models they all lead to the same conclusions in terms of maintenance decision. 
Some issues remain open and will be addressed in future works:

- The effects of the maintenance actions on the IFs different from Age have not been modelled. Inclusion of these aspects could be needed when assessing the performance of more complex maintenance policies.

- The case study considered is made up of a single component affected by only one degradation process. The potential of the framework needs to be tested on a multi-component and multidegradation processes system.

- The operation of defuzzyfication performed on the output of the Forward Module, does not propagate the uncertainties affecting the degradation state reached by the component. This leads to $M C$ simulations which sample from exponential distributions without considering the uncertainty of the parameters of those distributions.

- The fuzzy logic modelling framework has been developed by applying the Mamdani inference system [29], [30]. This limits the activation degrees of the degradation states to values smaller than 1 , i.e., it is not guaranteed that the maximum of the activation degree of the degradation state is equal to 1 . This problem, which leads to a smaller confidence on the degradation state, may be overcome by considering more sophisticated inference systems.

\section{Acknowledgment}

The authors wish to thank Eng. Guido Rossetti for his contribution to the work, within the development of his thesis at the Energy Department of the Politecnico di Milano.

\section{References}

[1]. Meeker W.Q., Escobar L.A.: Statistical Methods for Reliability Data, 1998. Wiley, N.Y.

[2]. Martorell S., Sanchez A., Serradell V.: Age-dependent reliability model considering effects of maintenance and working conditions. Reliability Engineering and System Safety, 1999, Vol. 64, pp. 19-31.

[3]. Singpurwalla N.D.: Survival in Dynamic Environment. Statistical Science, 1995, Vol. 10, No. 1, pp. 86-103..

[4]. Bogdanavičius V., Nikulin M.S.: Estimation in Degradation Models with Explanatory Variables. Lifetime Data Analysis, 2000, Vol.7, pp. 85-103.

[5]. Yashin, A., Arjas, E.: A note on random intensities and conditional survival functions. Journal of Applied Probability, 1988, Vol. 25, pp. 630-635.

[6]. Filkelstein, M.S.: On the exponential formula for reliability. IEEE Transactions On Reliability, 2004, Vol. 53-2, pp. 265-268.

[7]. Deloux E., Castanier B., Bérenguer C.: Predictive maintenance Policy for a gradually deteriorating system subject to stress. Reliability Engineering and System Safety, 2009, Vol. 94, pp. 418-431.

[8]. Jardine A.K.S., Ralston P., Reid N., Stafford J.: Proportional hazards analysis of diesel engine failure data, Quality and Reliability Engineering International, 1989, Vol. 5 pp. 207-16.

[9]. Zille V., Bérenguer C., Grall A., Despujols A., \& Lonchampt J.: Modelling and performance assessment of complex maintenance programs for multi-component systems. Proceedings of the 32nd ESReDA seminar and 1st ESReDA-ESRA seminar, 2007, pp. 127-140. Alghero, Italy. 
[10]. Baraldi P., Zio E., Compare M., Rossetti G. and Despujols A.: A novel approach to model the degradation of components in electrical production plants. Proceedings of the European Safety and Reliability Conference ESREL 2009, Praha, Czech Republic.

[11]. Baraldi P., Zio E., Compare M., Rossetti G. and Despujols A.: Modeling of degradation mechanisms in electrical components taking into account their actual living conditions. Proceedings of the International Conference in Reliability Maintenance and Safety ICRMS 2009. Chengdu, China.

[12]. Pham H., Wang H.: Imperfect Maintenance. European Journal of Operational Research, 1996, Vol. 139, pp. 469-489.

[13]. Clavareau J., Labeau P.E.: An Alternative imperfect preventive maintenance model. Safety Reliability and Risk analysis: Theory, Methods and Applications. Proceedings of Esrel 2008, Valencia (Spain). S. Martorell et al. Eds.

[14]. Clavareau J., Labeau P.E.: A model of imperfect preventive maintenance efficiency based on the concepts of elasticity and effective age. Safety Reliability and Risk analysis: Theory, Methods and Applications. Proceedings of Esrel 2009, Praha (Czech Republic). Briš et al. Eds.

[15]. Doyent L., Gaudoin O.: Classes of imperfect repair models based on reduction of failure intensity or virtual age. Reliability Engineering and System Safety, 2004, Vol. 84, pp. 45-56.

[16]. Castanier B., Bérenguer C., Grall A.: A sequential condition-based repair/replacement policy with non-periodic inspections for a system subject to continuous wea. Applied Stochastic Models in Business and Industry, 2003, Vol. 19, pp. 327-347.

[17]. Ponchet A., Fouladirad M., Grall A.: Imperfect condition-based maintenance on a finite time span for a gradually deteriorating system. Safety Reliability and Risk analysis: Theory, Methods and Applications. Proceedings of Esrel 2009, Praha (Czech Republic). Briš et al. Eds.

[18]. Barata, J., Guedes Soares C., Marseguerra M., Zio E.: Simulation modelling of repairable multi-component deteriorating systems for 'on condition' maintenance optimization. Reliability Engineering and System Safety, 2002, Vol. 76, pp. 255-264.

[19]. Nicolai R.P., Frenk J.B.G., Dekker R.: Modeling and optimizing imperfect maintenance of coatings on steel structures. Structural Safety,209, Vol. 31, pp. 234-244.

[20]. Zequeira R.I., Berenguer C.: Periodic Imperfect preventive maintenance with two categories of competing failure modes. Reliability Engineering and System Safety, 2006, Vol. 91, pp. 460-468.

[21]. Filkelstein M.S.: Wearing-out of components in a variable environment. Reliability Engineering and System Safety, 1999, Vol. 66, pp. 235-242.

[22]. Kumar D., Klefsjö B.: Proprotional hazard model A review. Reliability Engineering and System Safety, 1994, Vol. 44, pp. 177-188.

[23]. MIL-HDBK-217F. 1995, U.S. Department of Defense. ()

[24]. Vasely W.E.: Approaches for Age-Dependent Probabilistic Safety Assessment with Emphasis on Prioritization and Sensitivity Studies. NUREG/CR-5587,1992.

[25]. Marquis V., \& Solin J.: Fatigue design and Reliability. 1999, ESIS, Elsevier.

[26]. Shigley J. E., Mischke C. R., Brown, T. H.: Standard Handbook of Machine Design, Third Edition. 2004, Mc Graw-Hill.

[27]. Paris P. C., Erdogan F.: A critical analysis of crack propagation laws. Journal of Basic Engineering, Trans ASME, 1963, 85: 528-534.

[28]. Papoulis A., Pillai U.: Probability, Random Variables, and Stochastic Processes. $4^{\text {th }}$ Edition. 2002, Mc Graw-Hill

[29]. Mamdani E.H.: Application of fuzzy algorithms for control of a simple dynamic plant. Proceedings of the IEEE, 1974, 121(12), pp. 1585-1588. 
[30]. Babuska, R.: Fuzzy Modeling for Control, 1988, Kluwer Academic Publishers, Massachusetts. 